\title{
7-Hydroxycoumarin Elicit Anti-Angiogenic Effects Through Cellular Apoptosis in Developing Embryos of Zebrafish (Danio Rerio)
}

\author{
Narshimamurthy Anegundi \\ Katti Pancharatna \\ Department of Zoology, Karnatak University, Dharwad-580 003, India \\ doi: 10.19044/esj.2017.v13n21p53 URL:http://dx.doi.org/10.19044/esj.2017.v13n21p53
}

\begin{abstract}
Derivatives of coumarin have diverse biological activities and pharmacological significance. In this study we made an attempt to test one of the hydroxy derivative i.e. 7-hydroxycoumarin (7HC) for its anti-angiogenic potential using embryonic angiogenesis of zebrafish (Danio rerio) as biological model. Developing embryos of $D$. rerio were cultured in media containing one of the grade ( $1 \mathrm{mM}$ or $2 \mathrm{mM}$ or $3 \mathrm{mM}$ or $4 \mathrm{mM}$ or $5 \mathrm{mM}$ ) of concentration of $7 \mathrm{HC}$ from gastrula stage ( $\sim 6 \mathrm{hrs}$ post fertilization - hpf) until completion of embryonic development and hatching (72 hpf). Developmental trajectory of each post hatched larva was traced for two weeks. Angiogenesis and patterning of major blood vessels occurred in zebrafish embryos/ larvae between 24 - 72 hpf in controls. Exposure to 7HC resulted in an impairment in patterning of inter segmental vessels (ISVs) dorsal aorta (DA), dorsal longitudinal anastomotic vessel (DLAV), posterior cardinal vein (PCV), and common cardinal veins (CCVs) accompanied by increased site specific cellular apoptosis, declined heart rates in a dosedependent manner. 7HC manifest anti-angiogenic effects mediated/ induced through cellular apoptosis, hence may deserve attention and consideration as tumour angiogenesis suppressors in malignancy therapeutics.
\end{abstract}

Keywords: Zebrafish, Development, Embryo cultures, Angiogenesis, 7hydroxycoumarin

\section{Introduction}

Coumarins are phytochemicals found amply in fruits, vegetables, herbs and many plants. Derivatives of coumarin, are known to manifest variety of biological activities of medicinal value in treating human diseases and evolving therapeutics (Chen et al., 2004). For instance, many of the hydroxy derivative of coumarin, are used as an intermediate in the synthesis 
of anti-microbial, anti-inflammatory, anti-coagulant, and anti-proliferative agents (Ghate et al., 2005; Basanagouda et al., 2009; Moshal et al., 2011). Umbelliferone or 7-hydroxycoumarin (7HC) is hydroxy coumarin derivative which is widely used in the synthesis of drugs, protective agents, fluorescent probes and dyes etc., exhibits anti-oxidant, anti-diabetic, anti-inflammatory, anti-fungal, anti-proliferative properties (Gok, 2013; Lacy and Kennedy, 2004; Timonen et al., 2011). Scopoletin, a 7HC with a methoxy group at C6 position is used for the treatment of angiogenesis-mediated diseases clueing that synthetic analogues of this compound may have a pro or anti angiogenic potential (Pan et al., 2009). Angiogenesis is a process of sprouting of new blood plexuses from pre-existing blood vessels is a routine process during normal development but, has a special significance in tumorigenesis, as it supports and nourishes the tumor to grow from benign to malignant thereby augmenting metastasis. While, testing protocols for medicinal properties such as, anti-proliferative, anti-inflammatory, anti-bacterial of a chemical compound could easily be studied using cell lines, assessing angiogenic or anti-angiogenic value of a compound requires a biological model in which angiogenesis is actively in progress.

Zebrafish, has emerged as a powerful model system to understand human diseases and in evolving appropriate therapeutics. Developing embryos/ larvae of zebrafish are widely employed in testing the efficacy of a newly synthesized pharmaceutical or drug screening in medicine and cancer biology (Vascotto et al., 1997; Grunwald \& Eisen, 2002; Rubinstein, 2003; Amsterdam \& Hopkins, 2006; Tobia et al., 2011). Ex utero development, optical transparency and direct intake of chemicals/ drugs through skin have made zebrafish embryos/ larvae ideal for test protocols (Tobia et al., 2011). In this fish, formation of major blood vessels is initiated at $24 \mathrm{hpf}$ extends till 72 hpf (Isogai et al., 2003; Ellertsdóttir et al., 2010). The patterning of blood vessels of the zebrafish is distinct, in addition to major blood vessels (dorsal aorta - DA, dorsal longitudinal anastomotic vessel - DLAV and posterior cardinal vein - PCV) 26 pairs of inter-segmental vessels (ISVs), originate from dorsal aorta, run vertically and parallel to each other in between adjacent segments from trunk to tail that merge into the DLAV (Childs et al., 2002; Isogai et al., 2003; Moshal et al., 2011; Tobia et al., 2011; Gore et al., 2012). The common cardinal veins (CCVs) are formed at the anterior region of the trunk, where they collect the venous blood and transport it to the heart (Helker et al., 2013). In newly hatched semitransparent larvae (72 hpf) blood vessels are detectable and blood flow in them easily observable therefore, form ideal models/ test system for assessing the pro-angiogenic/ antiangiogenic property of a pharmaceutical (Tobia et al., 2011). The present study is an attempt to test whether $7 \mathrm{HC}$ exhibit anti-angiogenic activity in developing embryos of zebrafish. Live imaging, whole mount staining with 
alkaline phosphatase, O-dianisidine and acridine orange were carried out to trace patterning of blood vessels, path of blood flow and cellular apoptosis respectively. Cardiac anomalies (variation in size and pericardial edema) heartbeat rate, morphological abnormalities and rate of mortality were also recorded.

\section{Materials and Methods \\ Zebrafish}

Zebrafish (WT) were procured from Javed fisheries, Mumbai, India and maintained in the laboratory at $26 \pm 1{ }^{\circ} \mathrm{C}$ temperature and natural photo phase (11h dark and 13h light). Fish were fed twice a day on commercial pellets and Artemia nauplius ad libitum (Westerfield, 2007; Rajapurohit and Pancharatna, 2007). Dried tubifex worms were fed on alternate day. Permission to work on zebrafish was obtained from Animal Ethical Committee, CPCSEA, Government of India, under Institutional Registration \# 639/GO/02/a/CPCSEA.

\section{Breeding and raising embryos in Laboratory}

Gravid females and adult males in $1: 2$ ratio were placed in the breeding tank (in which a breeding trap made out of nylon mesh with a pore size of $3 \mathrm{~mm}$ was immersed) one hour after the last feeding of the day at 17.00 hrs. By next daybreak, numerous (200 - 300) fertilized eggs measuring $0.7-1 \mathrm{~mm}$ were found collected at the bottom of the aquarium. Eggs were carefully siphoned off into petri-plates $(20 \mathrm{~mm}$ diameter $)$ using Pasteur pipettes and washed thoroughly (2 - 3 times) with distilled water to get rid of dirt/ waste particles and then rinsed with E3 embryo medium. Healthy and embryos under development were selected under a stereo zoom microscope (Leica MZ6) for experimentation.

\section{Embryo cultures and exposure protocols}

7-hydroxycoumarin (Sigma-Aldrich, St Louis, USA) was dissolved in $0.5 \%$ DMSO (Dimethyl sulfoxide) (Qualigens, India) in double distilled water to get a stock solution. Exposure media (graded concentrations of 1 $\mathrm{mM}, 2 \mathrm{mM}, 3 \mathrm{mM}, 4 \mathrm{mM}$ and $5 \mathrm{mM}$ ) were prepared in $5 \mathrm{ml}$ of E3 embryo medium with $0.12 \mathrm{ml}$ of DMSO. A total of 60 embryos in triplicate sets $(n=20 /$ set $)$ were used for exposure to each concentration. Corresponding controls (exposed to $5 \mathrm{ml}$ of E3 medium with final concentration of $0.12 \mathrm{ml}$ of DMSO) were maintained. The duration of exposure was for a total of 68 hours starting from 06 - 72 hpf. After 24 hrs, 0.003\% 1-Pheny 2-thiourea (PTU) (Himedia, India) was added to the medium to minimize the development of pigmentation (Westerfield, 2007). At 72 hpf, embryos were hatched into larvae. The larvae were observed critically for patterning of 
blood vessels using alkaline phophatase staining (ALP), direction of blood flow/ RBC by whole mount staining for expression of globulin (Odianisidine) in RBC, caridiac structure and heart rates (beats per minute), cellular apoptosis (acridine orange) were recorded using Olympus bright field research microscope and images were captured (ProgRess 3 camera).

\section{Embryo (E3) medium and PFA Fixative}

E3 embryo medium was prepared for $60 \mathrm{X}$ stock solution $(17.2 \mathrm{~g}$ $\mathrm{NaCl}, 0.76 \mathrm{~g} \mathrm{KCl}, 2.9 \mathrm{~g} \mathrm{CaCl}_{2} .2 \mathrm{H}_{2} \mathrm{O}$ and $4.9 \mathrm{~g} \mathrm{MgSO}_{4} .7 \mathrm{H}_{2} \mathrm{O}$ were dissolved in $1 \mathrm{~L}$ double distilled water and stored at $5^{\circ} \mathrm{C}$ ). $16 \mathrm{ml}$ of $60 \mathrm{X}$ stock solution was diluted in $984 \mathrm{ml}$ of $\mathrm{dd}_{2} \mathrm{O}$ to get $1 \mathrm{X}$ stock solution to which $3 \mathrm{ml}$ of $0.01 \%$ methylene blue was added as fungicide (Westerfield, 2007; NussleinVolhard and Dahm, 2002).

4\% paraformaldehyde (PFA) was prepared by heating 4 g PFA in 50 $\mathrm{ml} \mathrm{dd} \mathrm{H}_{2} \mathrm{O}$ at $60^{\circ} \mathrm{C}$, to which 2 drops of $2 \mathrm{~N} \mathrm{NaOH}$ (to dissolve PFA), and 10 $\mathrm{ml} 10 \mathrm{X}$ PBS were added to make up the final volume $(100 \mathrm{ml})$ the $\mathrm{pH}$ of which was then adjusted 7.2 and stored at $2-4^{\circ} \mathrm{C}$ (Ausubel et al., 2003).

1X PBS (Phosphate Buffer Saline) was prepared by dissolving 136.8 $\mathrm{mM} \mathrm{NaCl}, 2.5 \mathrm{mM} \mathrm{KCl}, 0.8 \mathrm{mM} \mathrm{Na}_{2} \mathrm{HPO}_{4}, 1.47 \mathrm{mM} \mathrm{KH}_{2} \mathrm{PO}_{4}, 0.9 \mathrm{mM}$ $\mathrm{CaCl}_{2}$ and $0.5 \mathrm{mM} \mathrm{MgCl} 2 \cdot 6 \mathrm{H}_{2} \mathrm{O}$ in $800 \mathrm{ml} \mathrm{dd} \mathrm{H}_{2} \mathrm{O}$, the final volume was made to $1 \mathrm{~L}$ with $\mathrm{dd} \mathrm{H}_{2} \mathrm{O}$ and $\mathrm{pH}$ was adjusted to 7.4 and autoclaved (Ausubel et al., 2003).

\section{Whole-mount staining for endogenous alkaline phosphatase (ALP)}

At $72 \mathrm{hpf}$, the zebrafish embryos were processed for endogenous alkaline phosphatase (ALP) staining (Serbedzija et al., 1999; Moshal et al., 2011; Yeh et al., 2012). Embryos were fixed in 4\% PFA for $3.00 \mathrm{hrs}$, and incubated in $3 \% \mathrm{H}_{2} \mathrm{O}_{2}$ and $0.5 \% \mathrm{KOH}$ at room temperature for $30-60$ minutes until pigmentation disappears completely (Thisse and Thisse, 2008). Embryos were then washed 5-6 times in PBS (pH 7.4) with 0.1\% Tween-20 (Sigma-Aldrich, St Louis, USA) and subjected for dehydration and rehydration process (15 mins) in methanol (Himedia, India) - PBST gradation $(25 \%, 50 \%, 75 \%, 100 \%, 75 \%, 50 \%, 25 \%)$ and acetone for $30 \mathrm{~min}$ at $-20^{\circ} \mathrm{C}$ and finally suspended in PBST for $10-15 \mathrm{~min}$. These embryos were then equilibrated in alkaline phosphate (ALP) buffer with $100 \mathrm{mM}$ Tris $\mathrm{HCl}$ (pH 9.5) (Himedia, India), $50 \mathrm{mM} \mathrm{MgCl}, 100 \mathrm{mM} \mathrm{NaCl}$ and $0.1 \%$ Tween-20 for 45 mins followed by incubation in staining solution, 110 $\mu \mathrm{g} / \mathrm{mL}$ NBT (Nitro Blue Tetrazolium, Sigma-Aldrich, St Louis, USA) and $55 \mu \mathrm{g} / \mathrm{mL}$ BCIP (5-bromo-4-chloro-3-indolyl phosphate, Sigma-Aldrich, St Louis, USA) in alkaline phosphatase buffer at room temperature for $15-20$ min. The staining was terminated by using stop buffer which was prepared with $0.25 \mathrm{mM}$ EDTA (ethylene diamine tetra acetic acid) (Himedia, India) in 
PBST, pH 5.5 for 5 min. After completion of staining, embryos were fixed in $4 \%$ PFA overnight at $4{ }^{\circ} \mathrm{C}$, stored in $80 \%$ glycerol and were observed and photographed.

\section{Whole-mount staining for globin expression in RBC}

Whole-mount staining for globin expression in red blood cells (RBC) was peformed at $72 \mathrm{hpf}$ using O-dianisidine staining method (Iuchi and Yamamoto, 1983). Larvae were stained in dark (30 mins) in o-dianisidine $(0.6 \mathrm{mg} / \mathrm{ml}), 0.01 \mathrm{M}$ sodium acetate $(\mathrm{pH} 4.5), 0.65 \% \mathrm{H}_{2} \mathrm{O}_{2}$ and $40 \%$ ethanol. Stained larvae cleared in benzyl benzoate/ benzyl alcohol (2:1) and examined under Olympus brightfield research microscope and photographed (Detrich et al., 1995).

\section{Acridine Orange (AO) staining}

In order to check the cellular apoptosis induced if any, owing to drug exposure, larvae of both control and chemical exposed groups were stained at $72 \mathrm{hpf}$ with $5 \mu \mathrm{g} / \mathrm{mL}$ acridine orange dissolved in E3 medium for $15 \mathrm{~min}$ followed by 3 washings in the medium (David and Pancharatna 2009ab). They were then observed under a fluorescent microscope, the DNA intercalated $\mathrm{AO}$ fluoresces green at $525 \mathrm{~nm}$ (David and Pancharatna 2009ab).

\section{Heart rate}

In order to assess the changes if any in the appearance of heart and heart rate of experimental embryos/larvae associated with drug exposure, heartbeats per minute were $(\mathrm{bpm})$ recorded at $\left(26 \pm 1{ }^{\circ} \mathrm{C}\right)$ once in every 24 hour till day 7 using an inverted microscope Olympus IX71S8F. The Mean \pm S.E / group were calculated. Tricaine $(100 \mu \mathrm{g} / \mathrm{ml}$ in E3 media) (SigmaAldrich, St Louis, USA) was used to immobilize larvae (Schwerte et al., 2003).

\section{Statistical Analysis}

The data were analyzed using one-way analysis of variance (ANOVA) using SPSS (version 16.0), followed by Tukey's multiple comparison test. Statistical significance was defined at $\mathrm{P}<0.05$ level of significance. Percent inhibition of formation of blood vessels especially inter segmental blood vessels (ISVs) were determined for each drug by linear regression analysis of dose-response curve plotted for percent of inhibition of ISVs development against concentrations of drugs tested and IC50 (50\% inhibition) values were calculated. 


\section{Results}

\section{Controls}

The larvae of control group, underwent normal time-line development. Patterning of all major blood vessels i.e. DA, DLAV, ISVs and PCV was complete by 72 hpf. In newly hatched larvae of this group, a total of $26 \pm 0$ ISVs were found distributed from trunk to the tip of tail (Figs. 1A2). The development of heart and patterning of CCVs was normal (Fig. 3A). And RBCs were seen in all major blood vessels including ISVs (Fig. 3B). Heart rate varied from $91 \pm 1.02 \mathrm{bpm}$ to $160 \pm 1.45 \mathrm{bpm}$ from day 1 to day 7 (Fig. 4). No morphological abnormalities or cellular apoptosis were evident and all the 60 larvae survived up to 15 days (Fig. 5A, B and 6).
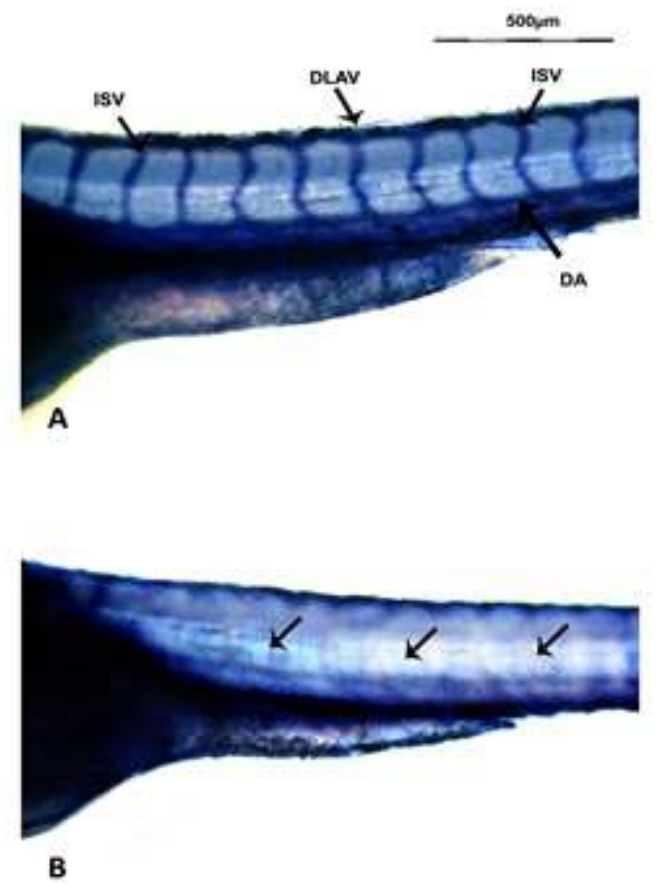

Fig. 1A-B. Trunk region of whole-mount stained D. rerio larvae (at $72 \mathrm{hpf}$ ) for endogenous alkaline phosphatase (ALP). (Scale bar $=500 \mu \mathrm{m})$.

Larva belonging to control group showing inter segmental vessels (ISV) and other major blood vessels (arrows) (A). Larva of embryo exposed to $5 \mathrm{mM} 7 \mathrm{HC}$ (B). Showing formation of incomplete ISVs (arrows).

(DA -Dorsal aorta, DLAV-Dorsal longitudinal anastomotic vessel, ISVs-Inter segmental vessels). 

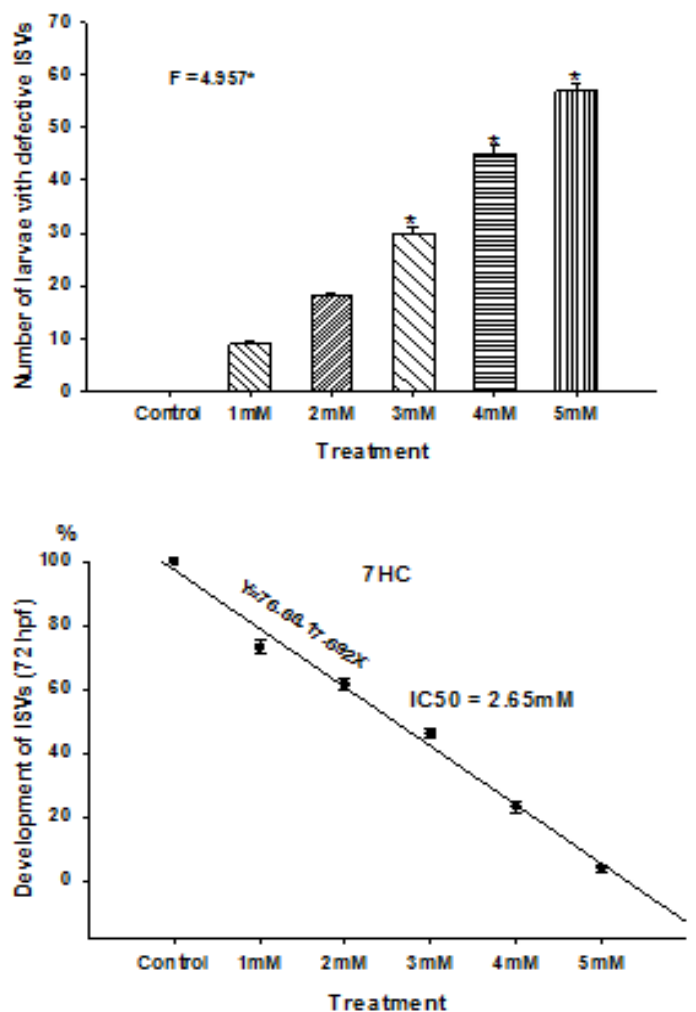

Fig. 2. Effects of 7HC on patterning of ISVs. IC50 Inhibitory concentration of chemical for $50 \%$ of ISV development. * Significant at $5 \%$ level.
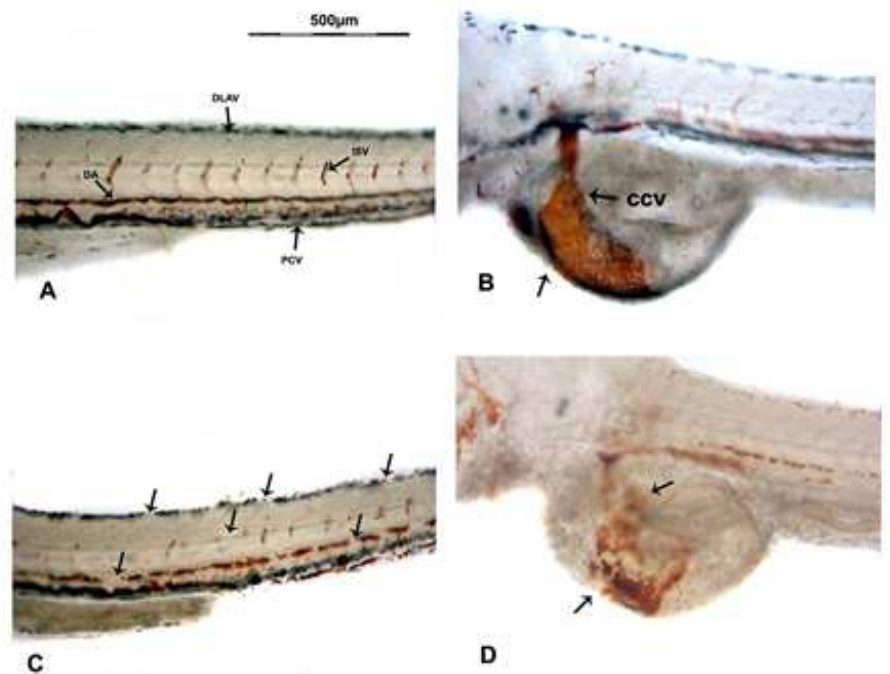

Fig. 3A-D. Trunk and heart regions of D. rerio larvae at $72 \mathrm{hpf}$ for globin expression in $\mathrm{RBC}$ (whole-mount staining for O-dianisidne). (Scale bar $=500 \mu \mathrm{m})$. 
Larva belonging to control group (A-B) showing ISVs, PCV, DA and DLAV (A) and Cardiac region and CCVs (arrows) (B). Larva of embryo exposed to 7HC (C- D) showing malformed ISVs and blood flow restriction in CCVs (arrows).

DA - Dorsal Aorta, DLAV-Dorsal longitudinal anastomotic vessel, ISVs-Inter segmental vessels and PCV-Posterior cardinal vein, CCVs-Common cardinal veins.

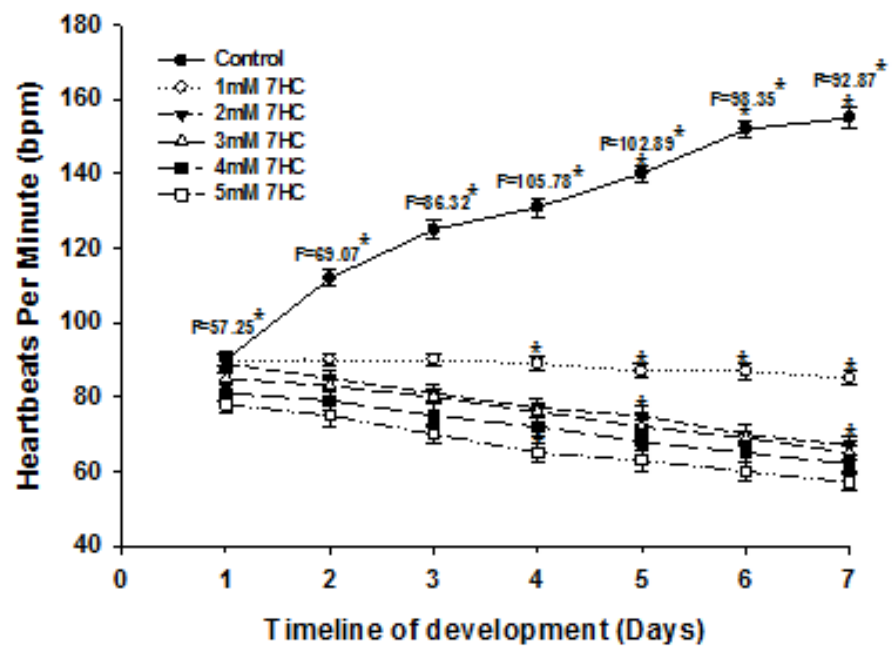

Fig. 4. Effects of $7 \mathrm{HC}$ on heart rate. * significant at $5 \%$ level.
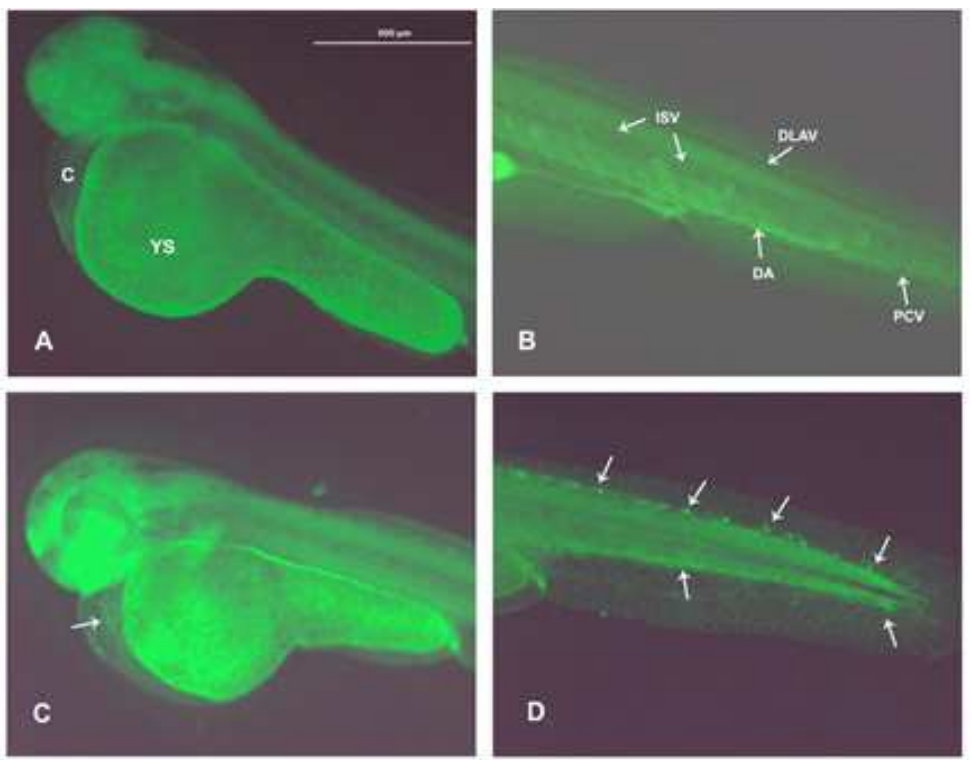

Fig. 5. Trunk and heart regions of zebrafish larvae (72 hpf) showing accumulation of positive cells (arrows) (Scale bar $=500 \mu \mathrm{m}$ )

(A-B) Larva belonging to control group.

(C-D) Larva of embryo exposed to $5 \mathrm{mM} 7 \mathrm{HC}$.

Arrows show AO - positive cells.

C - Cardiac region, YS - Yolk Sac, ISV - Inter segmental vessels, DLAV - Dorsal longitudinal anastomotic vessel, DA - Dorsal Aorta 

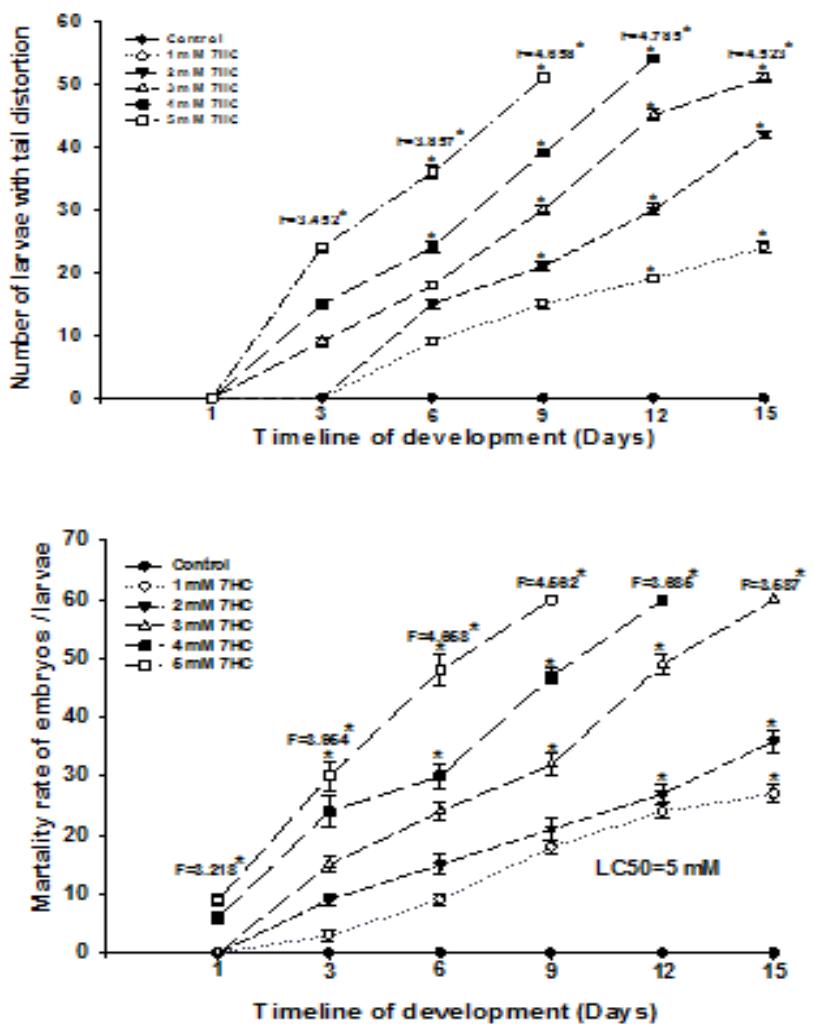

Fig. 6. Tail distortion and rate of mortality in larvae of embryo exposed to 7HC. * significant at $5 \%$ level.

Effects of exposure to graded concentrations of $7 \mathrm{HC}$ on the development

Figures $1-2$ show the suppression effects of $7 \mathrm{HC}$ on the formation of ISVs, number of larvae showing malformed ISVs and the impairment of blood flow in the larvae of treated groups as compared to controls. Twenty one out of 26 ISVs were defective in 9/60 larvae in $1 \mathrm{mM} 7 \mathrm{HC}$ exposure group, while, in $5 \mathrm{mM}$ concentration, 24/26 ISVs were found defective in $57 / 60$ as compared to all 26 well developed ISVs in all 60 control larvae (Figs. 1B-2). Overall, 7HC interfered ISVs formation with an average of $50 \%$ inhibition (IC50) value of $2.65 \mathrm{mM}$ (Fig. 2). RBC were observed in DA, DLAV, CCV, PCV and ISVs of control larvae but remained absent in ISVs or CCVs of $5 \mathrm{mM}$ concentration drug exposed larvae (Fig. 3C and D). Heart rates declined in all graded $7 \mathrm{HC}$ exposure groups in a dose dependent manner compared to those of controls (Figs. 4). Accumulation of AO positive cells was clearly visible in cardiac region and along DA, ISVs and DLAV of larvae of chemical exposed groups as compared to corresponding controls (Figs. 5C and D). The tail distortion was noticed in greater number 
of larvae and survival rate decreased as concentration of $7 \mathrm{HC}$ increased with LC50 value of $5 \mathrm{mM}$ (Fig. 6).

\section{Discussion}

Zebrafish cell lines, embryo cultures, and larvae are widely employed in drug screening research by pharmaceutical industries (Rubinstein, 2003;Parng, 2004; Pancharatna and David, 2008). The efficacy of a newly synthesized pharmaceutical or drug can be easily tested on zebrafish embryos/ larvae owing to their ready uptake of test compounds through chorion/ skin without involving complicated procedures for mode of administration (Sussman, 2001; Spitsbergen and Kent 2003; Rubinstein, 2003; Parng et al., 2004). Experimental studies on zebrafish embryos have demonstrated that many naturally occurring plant products/ metabolites promote or interfere important biological/ developmental pathways, for instance, the anti-angiogenic effects of cinnamon (Bansode et al., 2013), effects of caffeine on angiogenesis (Yeh et al., 2012) and protective effects of silymarin on curcumin intoxication (Shiau et al., 2011) etc. Earlier studies from our laboratory have reported that over the counter (OTC) pharmaceuticals (COX inhibitors, non steroidal anti-inflammatory drugs and anti ulcer drugs) have teratological implications and induce developmental anomalies zebrafish embryos/ larvae (Pancharatna and David, 2008; David and Pancharatna 2009ab).

The findings of our present study indicate that hydroxy derivative of coumarin (7HC) tested interfered with the patterning of major blood vessels thereby interrupting the blood flow in them. Standardized whole mount staining protocols for ALP as a marker for endothelial cells of sprouting blood vessels (Serbedzija et al., 1999; Moshal et al., 2011; Yeh et al., 2012), O-dionisidine for detecting RBC thereby the detection blood flow (Detrich et al., 1995) and AO staining for cells under apoptosis (Darzynkiewicz, 1990; David and Pancharatna 2009ab) were used. The angiogenesis suppressing activity of the drug $7 \mathrm{HC}$ was clearly evident by IC 50 value calculated (Fig. 2 ) and the mode of action was through increased cellular apoptosis was clear by the presence of AO positive cells at the regions of blood vessel sprouting suggesting explicitly that the cellular of action of drugs is via the enzymes that initiate apoptosis (Fig. 5). Earlier studies conducted in laboratory on malignant cell lines report that, coumarins especially $7 \mathrm{HC}$ is known to interfere cell cycle through apoptosis in human lung carcinoma cells (LopezGonzaleza et al., 2004) and HeLa cells (Chuang et al., 2007; Bronikowska et al., 2012). Osthole, a natural coumarin inhibits growth of hepatocellular carcinoma by the induction of apoptosis (Zhang et al., 2012). In this investigation, 7HC induced cellular apoptosis at cardiac region also as evidenced by the accumulation of AO positive apoptotic cells accompanied 
by decline in heart rates (Fig. 4-5). In addition, 7HC caused tail distortion and increased mortality at higher dose levels (with LC50 value of $5 \mathrm{mM}$ ).

Angiogenesis is known to play an important role in the growth and spread of tumour (Folkman and Shing, 1992; Folkman, 1995). Many naturally occurring and laboratory synthesized chemical compounds are demonstrated to interfere/ inhibit the angiogenesis and have therapeutic value in suppression of the tumour growth (Yeh et al., 2012; Bansode et al., 2013; Shiau et al., 2011). In our present study, we have demonstrated that in fish embryos 7HC manifest anti-angiogenic potential. This coumarin derivative have been already shown to exhibit properties such as, antitumour and anti-cancer on cell lines, and the present study is the first report to demonstrate anti-angiogenic property of this compound in embryonic angiogenic model. The results lead us to conclude that 7 hydroxy derivative of coumarin have anti-angiogenic potential and may form effective combination with other anti-neoplastic drugs in early cancer therapy.

\section{Acknowledgements}

This work was supported by UGC-SAP DSA-Phase I [F. 418/2015/DSA-I (SAP-II)] grant from New Delhi, India.

\section{References:}

1. Amsterdam, A. and Hopkins, N. (2006). Mutagenesis strategies in zebrafish for identifying genes involved in development and disease. Trends in Genetics, 22, 473-478.

2. Ausubel, F.M., Brent, R., Kingston, R.E., Moore, D.D., Seidman, J.G., Smith, J.A., Struhl, K. (2003). Short Protocols in Molecular Biology, $5^{\text {th }}$ Edition.

3. Bansode, R.R., Leung, T.C., Randolph, P., Williams, L.L. Ahmedna, M. (2013). Cinnamon extract inhibits angiogenesis in zebrafish and human endothelial cells by suppressing VEGFR1, VEGFR2, and PKC mediated MAP kinase. Food Science \& Nutrition. 1(1): 7482.

4. Basanagouda, M., Kulkarni, M.V., Sharma, D., Gupta, V.K., Pranesha, P., Sandhyarani and Rasal, V.P. (2009). Synthesis of some new 4-aryloxmethylcoumarins and examination of their antibacterial and antifungal activities. J. Chem. Sci. 121 (4):485-495.

5. Bronikowska, J., Szliszka, E., Jaworska, D., Czuba, Z.P. and Krol, W. (2012). The Coumarin Psoralidin Enhances Anticancer Effect of Tumor Necrosis Factor-Related Apoptosis-Inducing Ligand (TRAIL). Molecules. 17, 6449-6464.

6. Chen, S., Cho, M., Karlsberg, K., Zhou, D., and Yuan, Y.C. (2004). Biochemical and Biological Characterization of a Novel Anti- 
aromatase Coumarin Derivative. The journal of biological chemistry. Vol. 279, No. 46, Issue of November 12, pp. 48071-48078.

7. Childs, S., Chen, J., Garrity, D.M. and Fishman, M.C. (2002). Patterning of angiogenesis in the zebrafish embryo. Development 129, 973-982.

8. Chuang, J., Huang, Y., Lu, H., Ho, H., Yang, J., Li, T., Chang, N. and Chung, J. (2007). Coumarin Induces Cell Cycle Arrest and Apoptosis in Human Cervical Cancer HeLa Cells through a Mitochondria- and Caspase-3 Dependent Mechanism and NF- $\kappa \mathrm{B}$ Down-regulation. in vivo. 21: 1003-1010.

9. Darzynkiewicz, Z. (1990). Differential staining of DNA and RNA in intact cells and isolated cell nuclei with acridine orange. Methods Cell Biol. 33, 285-298.

10. David, A. and Pancharatna, K. (2009a). Developmental anomalies induced by non-selective COX inhibitor (Ibuprofen) in zebrafish (Danio rerio). Environmental Toxicology and Pharmacology. 27(3): 390-395.

11. David, A. and Pancharatna, K. (2009b). Effects of acetaminophen (paracetamol) in the embryonic development of zebrafish (Danio rerio). Journal of Applied Toxicology. 29 (7) 597 - 602.

12. Detrich, H.W., Kiera, M.W., Chan, F.Y., Barone, L.M., Yee, K., Rundstadler, J.A., Prat, S., Ransom, D. and Zon, L.I. (1995). Intraembryonic hematopoietic cell migration during vertebrate development. Proc. Natl. Acad. Sci. USA, Developmental Biology, Vol. 92, pp. 10713-10717.

13. Ellertsdóttir, E., Lenard, A., Blum, Y., Krudewig, A., Herwig, L., Affolter, M., Belting, H. G. (2010). Vascular morphogenesis in the zebrafish embryo. Developmental Biology. 341, 56-65.

14. Folkman, J., Shing, Y. (1992). Angiogenesis. J Biol Chem. 267:10931-10934.

15. Folkman, J. (1995). Angiogenesis in cancer, vascular, rheumatoid and other disease. Nat Med. 1:127-131.

16. Ghate, M., Kusanur, R.A. and Kulkarni, M.V. (2005). Synthesis and in vivo analgesic and anti-inflammatory activity of some biheterocyclic coumarin derivatives. Eur J Med Chem \& Res. 40(9):882-887.

17. Gök, E. (2013). Investigation of Binding Properties of Umbelliferone (7Hydroxycoumarin) to Lysozyme. J Fluoresc. 23:333-338.

18. Gore, A. V., Monzo, K., Cha, Y. R., Pan, W. and Weinstein, B. M. (2012). Vascular Development in the Zebrafish. Cold Spring Harb Perspect Med 2:a006684. 
19. Grunwald, D.J. and Eisen, J.S. (2002). Headwaters of the zebrafish emergence of a new model vertebrate. Nature Reviews Genetics 3, 717-724.

20. Helker, C.S.M., Schuermann, A., Karpanen, T, Zeuschner, D., Belting, H.G., Affolter. M., Stefan Schulte Merker, S and Herzog, W. (2013).The zebrafish common cardinal veins develop by a novel mechanism: lumen ensheathment. Development 140, 2776-2786.

21. Isogai, S., Lawson, N.D., Torrealday, S., Horiguchi, M. and Weinstein, B.M. (2003). Angiogenic network formation in the developing vertebrate trunk. Development and Disease. 130 (21), 5281-5290.

22. Iuchi, I., Yamamoto, M. (1983). Erythropoiesis in the developing rainbow trout, Salmo gairdneri irideus: histochemical and immunochemical detection of erythropoietic organs. J Exp Zool. 226: 409-417.

23. Lacy, A. and O'Kennedy, R. (2004). Studies on Coumarins and Coumarin-Related Compounds to Determine their Therapeutic Role in the Treatment of Cancer. Curren Pharmaceutical Design. 10, 3797-3811.

24. Lopez-Gonzaleza, J.S., Prado-Garciaa, H., Aguilar-Cazaresa, D., Molina-Guarnerosc, J.A., Morales-Fuentesb, J. and Mandokic, J.J. (2004). Apoptosis and cell cycle disturbances induced by coumarin and 7-hydroxycoumarin on human lung carcinoma cell lines. Lung Cancer. 43, 275-283.

25. Moshal, K.S., Ferri-Lagneau, K.F., Haider, J., Pardhanani, P. and Leung, T.C. (2011). Discriminating Different Cancer Cells Using a Zebrafish in Vivo Assay. Cancers. 2011, 3, 4102-4113.

26. Nusslein-Volhard, C. and Dahm, R. (2002). Zebrafish: A Practical Approach. Oxford University Press. 303p.

27. Pan, R., Dai, Y., Yang, J., Li, Y., Yao, X., Xia, Y. (2009). Antiangiogenic potential of scopoletin is associated with the inhibition of ERK 1/2 activation. Drug Dev Res. 70:214-219.

28. Pancharatna, K. and David, A. (2008). Developmental anomalies induced by Over-the-Counter (OTC) medications in Zebrafish, Danio rerio. Proc. Internatl. Conf. Biodiv. Conserv. \& Management. 339345.

29. Parng, C., Anderson, N., Ton, C., McGrath, P. (2004). Zebrafish apoptosis assays for drug discovery. In: The Zebrafish: Cellular and Developmental Biology, 2nd ed. Methods Cell Biol. 76, 75-85.

30. Rajapurohit, S.V. and Pancharatna, K. (2007). Development and hatching success of Zebrafish Danio rerio under laboratory conditions. J. Of Adv. Zool. 28(1): 21-25. 
31. Rubinstein, A.L. (2003). Zebrafish: From disease modeling to drug discovery. Current Opinion in Drug Discovery \& Development. 6(2):218-223.

32. Schwerte, T., Überbacher, D and Pelster, B. (2003). Non-invasive imaging of blood cell concentration and blood distribution in zebrafish Danio rerio incubated in hypoxic conditions in vivo. The Journal of Experimental Biology. 206, 1299-1307.

33. Serbedzija, G.N., Flynn, E. \& Willett, C.E. (1999). Zebrafish angiogenesis: A new model for drug screening. Angiogenesis. 3: $353 \pm 359$.

34. Shiau, R.J., Shih, P.C. and Wen, Y.D. (2011). Effect of silymarin on curcumin - induced mortality in zebrafish (Danio rerio) embryos and larvae. Indian Journal of Experimental Biology. 49:491-497.

35. Spitsbergen, J.M., and Kent, M.L. (2003). The state of the art of the zebrafish model for toxicology and toxicologic pathology research advantages and current limitations. Toxicol Pathol. 31: 62-87.

36. Sussman, R. (2001). Direct DNA Delivery into zebrafish embryos employing tissue culture techniques. Genesis. 31: 1-5. 2001.

37. Thisse, C. and Thisse, B. (2008). High resolution in situ hybridization on whole-mount zebrafish embryo. Nat. protoc. 3: $59-69$.

38. Timonen, J.M., Nieminen, R.M., Sareila, O., Goulas, A., Moilanen, L.J., Haukka, M., Vainiotalo, P., Moilanen, E., Aulaskari, P.H. (2011). Synthesis and anti-inflammatory effects of a series of novel 7-hydroxycoumarin derivatives. European Journal of Medicinal Chemistry. 46, 3845-3850.

39. Tobia, C., Sena, G.D. and Presta, M. (2011). Zebrafish embryo, a tool to study tumor angiogenesis. Int. J. Dev. Biol. 55: 505-509.

40. Vascotto, S.G., Beckham, Y. and Kelly, G.M. (1997). The zebrafish's swim to fame as an experimental model in biology. Biochemistry and Cell Biology. 75, 479-485.

41. Westerfield, M. (2007). THE ZEBRAFISH BOOK, A guide for the laboratory use of zebrafish Danio (Brachydanio) rerio. University of Oregon Press, Eugene 5th Edition.

42. Yeh, C.H., Liao, Y.F., Chang, C.Y., Tsai, J.N., Wang, Y.H., Cheng, C.C., Wen, C.C., and Chen, Y.H. (2012). Caffeine treatment disturbs the angiogenesis of zebrafish embryos. Drug and Chemical Toxicology. 35(4): 361-365.

43. Zhang, L., Jiang, G., Yao, F., He, Y., Liang, G., Zhang, Y., Hu, B., Wu, Y., Li, Y. and Liu, H. (2012). Growth Inhibition and Apoptosis Induced by Osthole, A Natural Coumarin, in Hepatocellular Carcinoma. PLoS ONE. Volume 7, Issue 5, e37865. 1-9. 